\title{
Lung Barrier Function in COVID-19?
}

\author{
T. K. Sivabakya ${ }^{1}$ (D) - G. Srinivas ${ }^{1}$ \\ Accepted: 21 July 2020 / Published online: 18 August 2020 \\ (C) Springer Nature Switzerland AG 2020
}

\begin{abstract}
The novel coronavirus COVID-19 appears to strike some people more intensely than others. Some people only experience mild symptoms while others require hospitalization and ventilation. With the virus becoming more prevalent day by day, it is not just the elderly, but even young people are falling seriously ill. Various researchers across the world state that specific cells in the nasal passages, intestines, and lungs may be more susceptible to the infection. Shifting the focus and research towards epithelium might provide new insight towards understanding COVID-19. This article is an overview of how epithelium permeability in COVID-19 may associate with comorbidities and other factors.
\end{abstract}

Keywords COVID - 19 . Epithelial barrier $\cdot$ Lung function · Mortality rate $\cdot$ Immune response $\cdot$ Inflammatory actions . Comorbidities $\cdot$ Epithelial permeability

\section{Epithelium and Immunity}

For decades, epithelial cells in innate immunity have been known to prevent the growth of bacterial and other microorganisms in the mucosal membrane [1]. Gong et al. [2] in his study shows that besides its mucociliary clearance feature, epithelial cells are now known to kill or neutralize microorganisms by producing many molecular families. Epithelial cells play a significant role in immune response regulation, inflammation, and host response. Although the role varies depending upon the pathogens and antigens, most of the diseases have a common pathology that includes marked epithelial cell activation in the upper airways or lower airways or both. Accumulating evidence by Schleimer et al. [3] suggests that epithelial cells are essential in initiating, regulating and maintaining the airway's innate and adaptive immune response.

This article is part of the Topical Collection on Covid-19

T. K. Sivabakya

sivasyd.nov7@yahoo.com

G. Srinivas

srinivas.g@tnmgrmu.ac.in

1 Department of Epidemiology, The Tamilnadu Dr MGR Medical University, No.69, Annasalai, Guindy, Chennai 600032, India

\section{Pathogenesis of SARS-CoV-2}

The inhaled SARS-CoV-2 virus likely begins the replication on attachment to the epithelial cells in the nasal cavity. Angiotensin-converting enzyme 2 (ACE2) is the primary receptor for SARS-CoV2 and SARS-CoV. The virus spreads locally but has a minimal innate immune response. At this point, nasal swabs will detect the virus $[4,5]$. These individuals are infectious although the viral burden may be low. Clinically manifested at this time is the COVID-19 disease. Viral epithelial infected cells are an important source of beta and lambda interferons [6]. Determining the innate immune response of the host may improve predictions of the disease's subsequent course, and may require more aggressive monitoring. The disease would be mild for about $80 \%$ of the infected patients and mainly confined to the upper and conductive airways. Unfortunately, about $20 \%$ of the patients infected will advance to stage 3 disease and develop pulmonary infiltration, some of which will develop a very serious disease. So, the entire process starts when the virus infects the epithelial cells $[7,8]$.

\section{Mortality in COVID-19}

Based on the evidence and professional experience presently available, COVID-19 presents a greater risk for the elderly and individuals of any age who have specific underlying medical conditions. Older adults and those with chronic medical 
conditions, including cancer, heart disease, diabetes, lung disease, and hypertension are at higher risk of experiencing more serious complications accelerated by COVID-19. As the number of COVID-19 infections continues to rise, a curious scenario has begun to emerge of otherwise healthy people succumbing to the virus. Experts are unable to determine clearly why the virus was fatal to some of the youngest patients, but has only led to mild symptoms in others. Mounting research suggests that most extreme cases are caused by aberrant immune responses and are not reliant on viral loads. This is where the epithelial cells should be considered an important mediator of the destructive process.

\section{Epithelium and High-Risk Group}

A brief overview of the epithelium for people with underlying diseases can provide us with various insights on COVID-19. Diabetes is coupled with increased glucose that distresses the respiratory epithelium, or vice-versa [9]. Similarly, several reports show increased epithelial expression of matrix metalloproteinases (MMPs) in lung tissue of COPD (chronic obstructive pulmonary disease) patients, demonstrating a role for the epithelial cell in alveolar destruction and airspace enlargement [10]. Any type of cancer starts its mutation or proliferation from the epithelial cells. In asthma, impaired epithelial proliferation is suggested to cause the bronchial epithelium which leads to increased secretion of profibrogenic growth factors [11]. Thus, the epithelium becomes damaged due to its underlying comorbidities. This may let the SARS-Cov-2 virus easily attach to the permeable epithelium.

\section{Epithelium and Low-Risk Group}

However, in the lower risk younger population, the epithelium permeability may be associated with alcohol intake, smoking, or stress. Long-term exposure to alcohol reduces the ability of the epithelial barrier to stand up against infection. Acute exposure to $0.2 \%$ or higher concentrations of ethanol has been shown to adversely affect epithelial cell interactions. It also reduces close junctions in epithelia and adheres to them [12, 13]. During their lifetime, mucosal epithelia encounter both physicochemical and biological stress. Several mechanisms have evolved to deal with stress, including regulation of immune cell functions. Also, numerous studies prove that psychological stress is associated with intestinal epithelial hyperpermeability [14-16]. Animal studies have shown that chronic and sporadic tobacco smoke exposure induces morphological alterations of the entire respiratory tract from hyperplasia to the epithelium [17]. This provides us an insight of the epithelium barrier interaction amongst those people without underlying comorbidities.

\section{Epithelium Activation}

The mucosal immune system is composed of locally synthesized polymer IgA. The polymers bind to the immunoglobulin receptors present on the basolateral surface of the mucosal epithelial cells. The polymer IgA receptor mediates transcytosis and endocytosis. $\operatorname{IgA}$ functions in host defense at three levels relative to the mucosal epithelium [18]. The epithelial cells influence the $\mathrm{T}$ and $\mathrm{B}$ cells by releasing chemokines. The epithelial cells also control the proliferation, activation, differentiation, and survival of $\mathrm{T}$ and $\mathrm{B}$ lymphocytes. The ability to resolve viral lung infections depends on interferon (IFN) and inflammatory cytokine actions of the epithelium. Epithelial activation is a characteristic feature of rhinitis, rhinosinusitis, a chronic obstructive pulmonary disorder, asthma, and other airway diseases. The inability of the epithelium to maintain a physical and immunological barrier may play a role in its susceptible nature to the diseases [19, 20].

\section{Discussion}

The capacity to overcome lung viral infections relies on interferon (IFN) and inflammatory cytokine activities, but their respective contributions to host protection and return to homeostasis remain unknown. Type III IFNs (IFN- $\lambda$ ) in particular have received a great deal of interest as they work mainly on mucosal surfaces [21]. Previous studies revealed that IFN- $\lambda$ defends against viral infections and enhances the membrane roles of both intestinal epithelial cells and endothelial cells. Such differences can be clarified by the fact that in some experiments, the particular categories of cells attacked by IFN- $\lambda$ were different. In fact, in a recent study finding by Broggi et al. [22], they reinforce the theory that IFN- $\lambda$ adverse behavior happens even after prolonged contact and tissue harm exists. Perhaps, the early administration of IFN $-\lambda$ in a COVID-19 mouse model conferred safety.

\section{Conclusion}

Considering that the epithelium provides essential host protection, it is rational to enhance their role during infection to improve outcomes. As suggested by Sharma et al. [23], strengthening the epithelial host defense can provide an important immunomodulatory strategy. Watching over epithelial immunity not only prevents collateral damage that can be caused by recruited immune cells but can also avoid the need to suppress these immune cells from the tissue [23]. Improving our understanding of epithelial functioning under normal and pathological conditions might provide new insights. There must be consistency in the system and there 
may be more than one pathway. As we move forward in this field, novel pathways and factors will likely surprise us. The main focus will now be on supporting more in vitro or in vivo studies on this.

\section{Compliance with Ethical Standards}

Conflict of Interest The authors declare that they have no competing interests.

Ethical Approval Not applicable.

Informed Consent Not applicable.

\section{References}

1. Cookson W. The immunogenetics of asthma and eczema: a new focus on the epithelium. Nat Rev Immunol. 2004;4:978-88.

2. Gong JL, McCarthy KM, Telford J, Tamatani T, Miyasaka M, Schnee-berger EE. Intraepithelial airway dendritic cells: a distinct subset of pulmonary dendritic cells obtained by microdissection. J Exp Med. 1992;175:797-807.

3. Schleimer RP, Kato A, Kern R, Kuperman D, Avila PC. Epithelium: at the interface of innate and adaptive immune responses. J Allergy Clin Immunol. 2007;120(6):1279-84. https:// doi.org/10.1016/j.jaci.2007.08.046.

4. Wan Y, Shang J, Graham R, et al. Receptor recognition by novel coronavirus from Wuhan: an analysis based on decade-long structural studies of SARS. J Virol. 2020;94:e00127-0.

5. Hoffmann M, Kleine-Weber H, Schroeder S, Krüger N, Herrler T, Erichsen S, et al. SARS-CoV-2 cell entry depends on ACE2 and TMPRSS 2 and is blocked by a clinically proven protease inhibitor. Cell. 2020; in press;181:271-280.e8. https://doi.org/10.1016/j.cell. 2020.02.052

6. Tang NL, Chan PK, Wong CK, et al. Early enhanced expression of interferon-inducible protein-10 (CXCL-10) and other chemokines predicts adverse outcome in severe acute respiratory syndrome. Clin Chem. 2005;51:2333-40. https://doi.org/10.1373/clinchem. 2005.054460.

7. Wu Z, McGoogan JM. Characteristics of and important lessons from the coronavirus disease 2019 (COVID-19) outbreak in China: summary of a report of 72314 cases from the Chinese Center for Disease Control and Prevention. JAMA. 2020;323: 1239. https://doi.org/10.1001/jama.2020.2648.

8. Mason RJ. Pathogenesis of COVID-19 from a cell biology perspective. Eur Respir J. 2020;55(4):2000607. Published 2020 Apr 16. https://doi.org/10.1183/13993003.00607-2020.

9. Bano S, Swati O, Kambadur M, Mohammad F. Deterioration of epithelium mediated mechanisms in diabetic-antigen sensitized airways of Guinea pigs. J Smooth Muscle Res. 2016;52(0):93-104. https://doi.org/10.1540/jsmr.52.93.
10. Mercer BA, Lemaitre V, Powell CA, D'Armiento J. The epithelial cell in lung health and emphysema pathogenesis. Curr Respir Med Rev. 2006;2(2):101-42. https://doi.org/10.2174/157339806776843085.

11. Keller AC, Rodriguez D, Russo M. Nitric oxide paradox in asthma. Mem Inst Oswaldo Cruz. 2005;100(Suppl 1):19-23. https://doi. org/10.1590/s0074-02762005000900005.

12. Elamin E, Jonkers D, Juuti-Uusitalo K, van Ijzendoorn S, Troost F, Duimel $\mathrm{H}$, et al. Effects of ethanol and acetaldehyde on tight junction integrity: in vitro study in a three dimensional intestinal epithelial cell culture model. PLoS One. 2012;7(4):e35008. https://doi. org/10.1371/journal.pone.0035008.

13. Simet SM, Wyatt TA, DeVasure J, Yanov D, Allen-Gipson D, Sisson JH. Alcohol increases the permeability of airway epithelial tight junctions in Beas-2B and NHBE cells. Alcohol Clin Exp Res. 2012;36(3):432-42. https://doi.org/10.1111/j.1530-0277.2011. 01640.x.

14. Zhang L, Song J, Bai T, Qian W, Hou XH. Stress induces more serious barrier dysfunction in follicle-associated epithelium than villus epithelium involving mast cells and protease-activated receptor-2. Sci Rep. 2017;7:4950. https://doi.org/10.1038/s41598-01705064-y.

15. Duffy LC, Zielezny MA, Marshall JR, et al. Relevance of major stress events as an indicator of disease activity prevalence in inflammatory bowel disease. Behav Med (Washington, DC). 1991;17(3): 101-10. https://doi.org/10.1080/08964289.1991.9937553.

16. Garrett VD, Brantley PJ, Jones GN, McKnight GT. The relation between daily stress and Crohn's disease. J Behav Med. 1991;14(1):87-96. https://doi.org/10.1007/bf00844770.

17. Thorley AJ, Tetley TD. Pulmonary epithelium, cigarette smoke, and chronic obstructive pulmonary disease. Int J Chron Obstruct Pulmon Dis. 2007;2(4):409-28.

18. Lamm ME. Current concepts in mucosal immunity. IV. How epithelial transport of IgA antibodies relates to host defense. Am J Phys. 1998;274(4):G614-7. https://doi.org/10.1152/ajpgi.1998. 274.4.g614.

19. Turula $\mathrm{H}$, Wobus CE. The role of the polymeric immunoglobulin receptor and secretory immunoglobulins during mucosal infection and immunity. Viruses. 2018;10(5):237. Published 2018 May 3. https://doi.org/10.3390/v10050237.

20. Floege J, Feehally J. The mucosa-kidney axis in IgA nephropathy. Nat Rev Nephrol. 2016;12:147-56. https://doi.org/10.1038/nrneph. 2015.208.

21. Broggi A, Granucci F, Zanoni I. Type III interferons: balancing tissue tolerance and resistance to pathogen invasion. J Exp Med. 2020;217(1):e20190295. https://doi.org/10.1084/jem.20190295.

22. Broggi A, Ghosh S, Sposito B, Spreafico R, Balzarini F, Lo Cascio $\mathrm{A}$, et al. Type III interferons disrupt the lung epithelial barrier upon viral recognition. Science. 2020:eabc3545. https://doi.org/10.1126/ science.abc3545.

23. Sharma L, Feng J, Britto CJ, Dela Cruz CS. Mechanisms of epithelial immunity evasion by respiratory bacterial pathogens. Front Immunol. 2020;11:91. Published 2020 Feb 11. https://doi.org/10. 3389/fimmu.2020.00091.

Publisher's Note Springer Nature remains neutral with regard to jurisdictional claims in published maps and institutional affiliations. 